\title{
Isolation and biochemical characterization of Streptococus pneumoniae from throat swabs and sputum of children and adult in Quetta District
}

\author{
Rozina Rasheed ${ }^{1}$, Khalil Hasni2*, Muhammad Kamran Taj ${ }^{3}$, Muhammad \\ Shafi $^{3}$ and Shazia Rasheed ${ }^{2}$ \\ 1. Microbiology Department University of Balochistan-Pakistan \\ 2. Lasbella University of Agriculture, Water and Marine Sciences, Uthal, Balochistan-Pakistan \\ 3. Centre for Advanced Studies in Vaccinology and Biotechnology, University of Balochistan, Quetta-Pakistan \\ *Corresponding author's email: Khalil_hasni1@yahoo.com \\ Citation \\ Rozina Rasheed, Khalil Hasni, Muhammad Kamran Taj, Muhammad Shafi and Shazia Rasheed. Isolation and \\ biochemical characterization of Streptococus pneumoniae from throat swabs and sputum of children and adult in \\ Quetta District. Pure and Applied Biology. Vol. 9, Issue 3, pp1755-1762. \\ http://dx.doi.org/10.19045/bspab.2020.90186
}

Received: $17 / 01 / 2020 \quad$ Revised: 22/03/2020

Accepted: 04/04/2020

Online First: 13/04/2020

\section{Abstract}

The human pathogen Streptococcus pneumoniae is one of the main causative agents of respiratory tract infections. This study aims to isolate the $S$. pneumoniae from children and adults affected from throat infections in different hospitals in Quetta District. The sputum samples and throat swabs were collected from the adults and children suspected for suffering from respiratory tract infection on the source of clinical symptom. When the samples were analyzed on Columbia blood agar plates, among (120) samples, (40) isolates produced 2-1 mm in diameter alpha hemolytic colonies. In fresh culture the colonies were raised but later became flattened with raised edges, giving them a ringed appearance. Alpha hemolysis was produced underneath and zone was narrow around colonies on Columbia blood agar following incubation at $37^{\circ} \mathrm{C}$ in 5 10\% CO2. On selective media; Crystal Violet Nalidixic Acid Gentamycin (CVNG) blood agar all (40) isolates produced small, flat, draughtsman type colonies with greenish alpha hemolysis. When the colonies from CVNG were streaked on MacConkey agar, no growth had appeared. $S$. pneumoniae (pneumococcus) is an essential pathogen of human that causes both delicate upper respiratory infections and serious invasive infections like septicaemia, meningitis and pneumonia. The proper management is important for the treatment of pneumonia patients.

Keywords: Characterization; District; Pneumoniae; Quetta; Streptococus

\section{Introduction}

S. pneumoniae cells are lancet-shaped and gram-positive. Usually they are seen as pairs of cocci (diplococci) but some time occur singly and in short chains. The cells are between 0.5 and $1.25 \mathrm{~mm}$ in diameter. They 
are non-motile and do not form spores. It is facultative anaerobic bacteria and growing best in $5-10 \%$ carbon dioxide. Nearly $20 \%$ of fresh clinical isolates need fully anaerobic situation and in all cases, growth needs a source of catalase (e.g. blood) to deactivate the huge amount of hydrogen peroxide created by the bacteria. S. Pneumoniae doubling time is 20-30 minutes [1]. The most favorable temperature for growth is $37^{\circ} \mathrm{C}$ but it ranges from $25-40^{\circ} \mathrm{C}$. The organism die quickly in cultures e.g. in the course of a day or two, particularly in aerobic cultures in media without blood. The dead organisms tend to undergo autolysis. Thus an aerobic culture in shallow nutrient broth may be uniformly turbid after 6 to 12 hour and become clear by autolysis within $24 \mathrm{hr}$. On blood agar, S. Pneumoniae produce alpha (green) hemolysis, which differentiates $S$. Pneumoniae from the group A (beta hemolytic) streptococcus [1]. On blood agar colonies are transparent, small, smooth, flattened and depressed centrally showing the draughtsman form. The pneumonia is caused by a variety of bacteria including Streptococcus, Pseudomonas, Staphylococcus, Chlamydia, Hemophilic, Mycoplasma, Fungi, Viruses and Protozoans [2].

In medical microbiology $S$. Pneumoniae is known as the pneumococcus, referring to its shape and its regular involvement in pneumococcal pneumonia [1]. Itis the most commonly detected causative agent of community-acquired pneumonia which is well recognized as being potentially severe. Among immune-competent and immunesuppressed hosts, S. Pneumoniaeis readily recognized as a frequent cause of community-acquired pneumonia. The occurrence of pneumococcal disease is peak among children and the elderly. However respiratory infections are a key cause of mortality and morbidity among youngsters [3]. In children the $S$. Pneumoniae is a main causative agent of such infections [4]. The core ecological reservoir of $S$. Pneumoniae is nasopharynx, from where it can grow to disease after extending to other parts of the respiratory tract or penetrating normally sterile body fluids [5].

S. pneumoniae is a normal inhabitant of the human upper respiratory tract. The bacterium can cause paranasal sinusitis, pneumonia, and meningitis which is generally secondary to one of the former infections. S. Pneumoniae also causes endocarditis, osteomyelitis, septic arthritis, cellulitis, peritonitis and brain abscesses. They cause septicaemia in a proportion of these infections and it is particularly the septicaemic infections that may prove. The objective of study was to isolate and identify $S$. Pneumoniae to the sputum and throat swab samples from children and adults having throat infection in Quetta District.

Pneumonia infection is recognized as a major cause of morbidity and mortality in the community. Since an etiological diagnosis of pneumonia infection with susceptibility results is almost never available to assist in the selection of the prompt therapy, necessary for pneumonia infection, the initial approach to the treatment is largely empirical and is ordinarily guided by the risk category of the patient. The knowledge of predominant microbial patterns, however, provides at least as important basis or initial decisions about empirical therapy of pneumonia infection. The issues mentioned highlight the need for continuing the research at national and local level to attain effective therapy of pneumonia infection, as well as to promote rational prescribing of antimicrobials with consequent slowing of the development of resistance to both existing and new agents. In this light, to do away with the lack of authentic data on the etiology of pneumonia infection in Quetta Balochistan, this study aimed at 
investigating in a prospective study the spectrum and susceptibility ofStreptococcus pneumoniae of pneumonia infection and establishing associations with known clinical and demographic risk factors in population referred to a distinct hospital of Quetta city.

\section{Material and methods}

Present research was conducted at (CASVAB) Centre for Advanced Studies in Vaccinology and Biotechnology University of Balochistan, Quetta.

\section{Media and biochemical reagent preparation}

These following media and reagents were used for primary isolation of $S$. Pneumoniae. Columbia agar blood agar, Crystal violet nalidixic acid gentamycin (CVNG) agar, MacConkey agar, Triple sugar iron agar (TSI), Sulfide Indole Motility (SIM) medium, Sugar base medium, Bile salt, $1 \mathrm{~N}$ (Normality) $\mathrm{HCl}, 1 \mathrm{~N}$ (Normality) $\mathrm{NaOH}$.

All of media that are commercially available were prepared according to the manufacturing directions and autoclaved at $15 \mathrm{lb} / \mathrm{in}^{2}$ pressure per square inch (PSI) at $121^{\circ} \mathrm{C}$ for 15 minutes. Then allowed it to cool down up to $50^{\circ} \mathrm{C}$ or filtered as per requirement.

The liquid media were added in sterile test tubes having cotton plugs. The solid fresh media were aseptically put into plates and in pre-sterile tubes for slant preparations and were allowed for solidification. These plates and tubes of media were incubated for $24 \mathrm{hr}$ at $37^{\circ} \mathrm{C}$ in order to check sterility of the media. In case any media showed fungal or bacterial growth were discarded. Sterile media was stored at $4{ }^{\circ} \mathrm{C}$ for further processing.

\section{Collection of samples}

Throat swabs and sputum samples were collected from outdoor patients (children and adults) of Bolan Medical Complex (BMC) Hospital and Provincial Sandeman Hospital (PSH) in a pre-sterile pre-labeled, dry, wide-necked, leak proof container, care were taken in order to prevent, spread of infectious organisms, and also to avoid contamination the outside of the container. Swab samples were collected by swabbing the affected area by using a sterile cotton wool swab and then immediately returned the swab to its sterile containers. Before sample taken from patients; for 8 hours before swabbing and taking sputum, the patient should be not treated with any kind of antibiotics or antiseptic mouths wash [6].

\section{Transport of samples}

The throat swabs and sputum samples were transported to laboratory in cooled chain condition within 1hour, while sputum samples were diluted in $5 \mathrm{ml} \mathrm{NaCl}$ in the container during transportation [6].

\section{Sample processing}

Collected samples were streaked on Columbia blood agar plates. The colonies having alpha hemolytic were picked up and then restreaked on Columbia blood agar plates in order to obtain pure bacterial colonies. For further confirmation of Streptococcus pneumonia purified growth of alpha hemolytic colonies were streaked on selective medium crystal violet nalidixic acid gentamycin (CVNG) blood agar plates. Then pure colonies from CVNG blood agar plate were further processed for confirmation through gram staining and biochemical tests (catalase, oxidase, optochin disc test, bacitracin disc, different sugars fermentation test).

\section{Results}

\section{Growth on columbia blood agar}

Samples were streaked on Columbia Blood agar. Following incubation, the plates were observed for growth. The colonies were appeared around 1-2 $\mathrm{mm}$ in diameter. In fresh culture plates the colonies were raised but later on it became flattened with raised their edges that gave them a ringed like appearance. Pneumococcus produced alpha heamolytic colonies as shown in (Fig 1). 
Growth on crystal violet nalidixic acid gentamycin agar (CVNG)

Colonies from Columbia blood agar were streaked on CVNG agar. After incubation, plates were examined. Following incubation, colonies appeared as small, flat, draughtsman type with greenish alpha haemolysis as shown in (Fig. 2).

\section{Growth on maconkey agar}

Colonies from selective medium were streaked on MacConkey agar. When the plates of MacConkey agar were examined after incubation, no growth had appeared. As S. Pneumoniaecannot grow on MacConkey agar shown in (Fig 3). MacConkey agar inhibits the growth of Gram positive bacteria.

\section{Gram staining}

Organisms were found as violet color, elongated (lancet-shaped) mostly diplococcus. Most of them formed short chains giving Gram positive staining reaction as shown in (Fig. 4).

\section{Determination of pathogenicity on blood agar}

On the Blood agar plates the isolated colonies were streaked and then incubated for 24 hours at $37^{\circ} \mathrm{C}$ in $5-10 \% \mathrm{CO} 2$, in order to determine its potential ability to lyse the sheep erythrocytes (RBCs). After incubation, colonies were observed for heamolysis. The hemolytic positive strains appeared partially clearing of the blood and also greenish discoloration due to (alpha hemolysis) were created beneath in a narrow zone in and around the bacterial colonies as shown in (Fig 5).

\section{Biochemical tests}

Citrate test, sugar fermentation test (Glucose, TSI and Raffinose), Oxidase test, Bile solubility test, $6.5 \% \mathrm{NaCl}$ test and CAMP tests were performed in order to confirm and identify the isolated bacteria as shown in (Table 1).

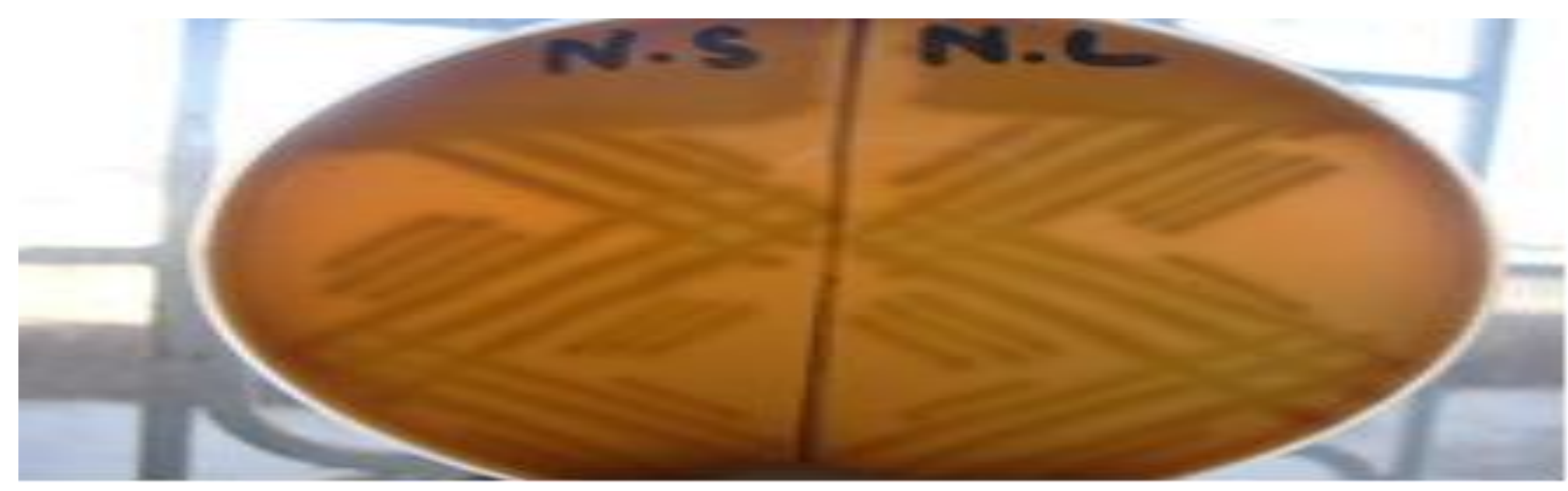

Figure 1. Colonies of S. Pneumoniae on Columbia blood agar

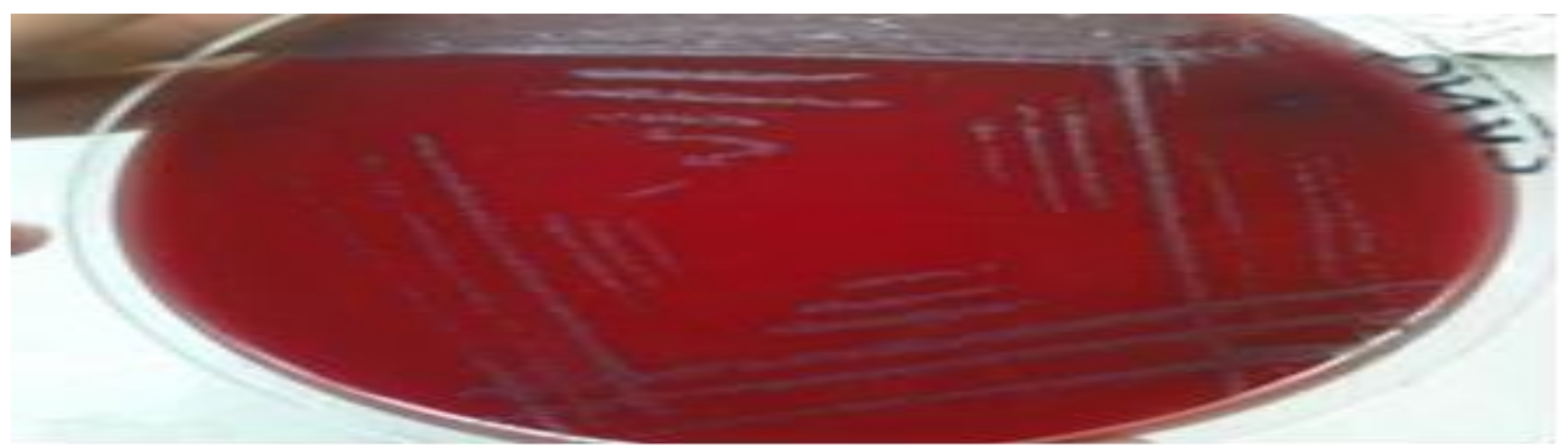

Figure 2. Colonies of S. Pneumoniae on CVNG medium 


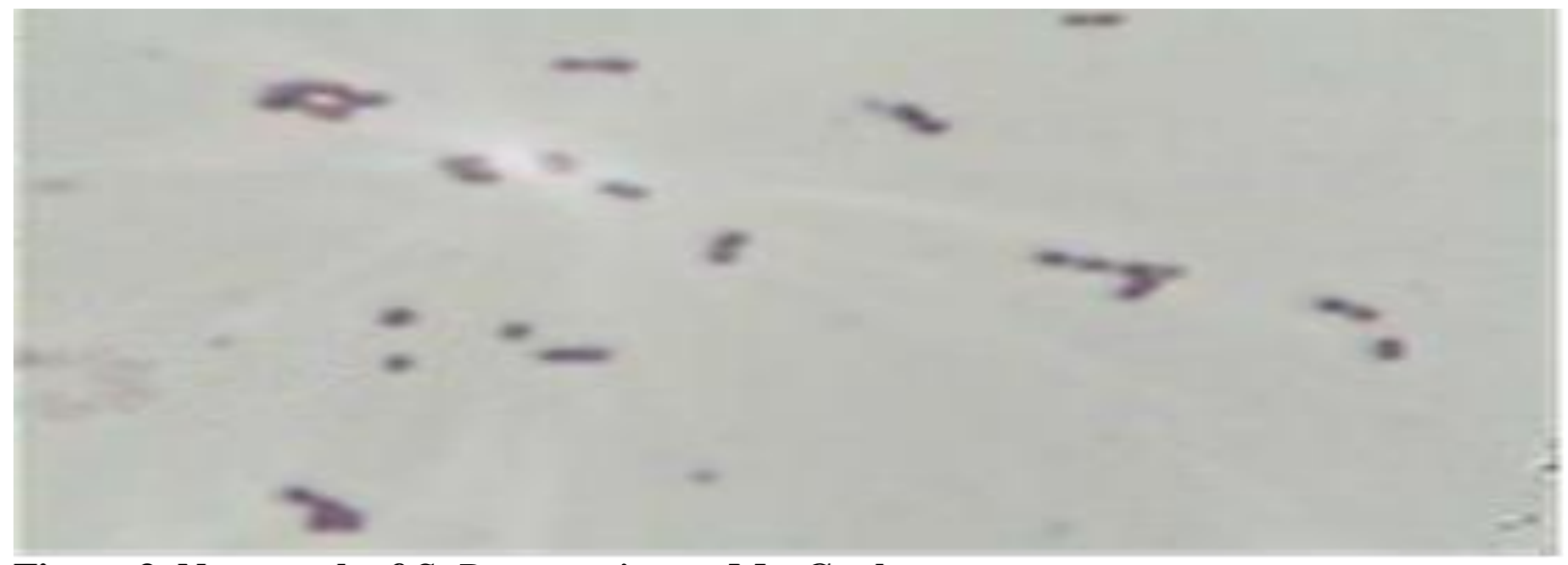

Figure 3. No growth of S. Pneumoniae on MacConkey aga

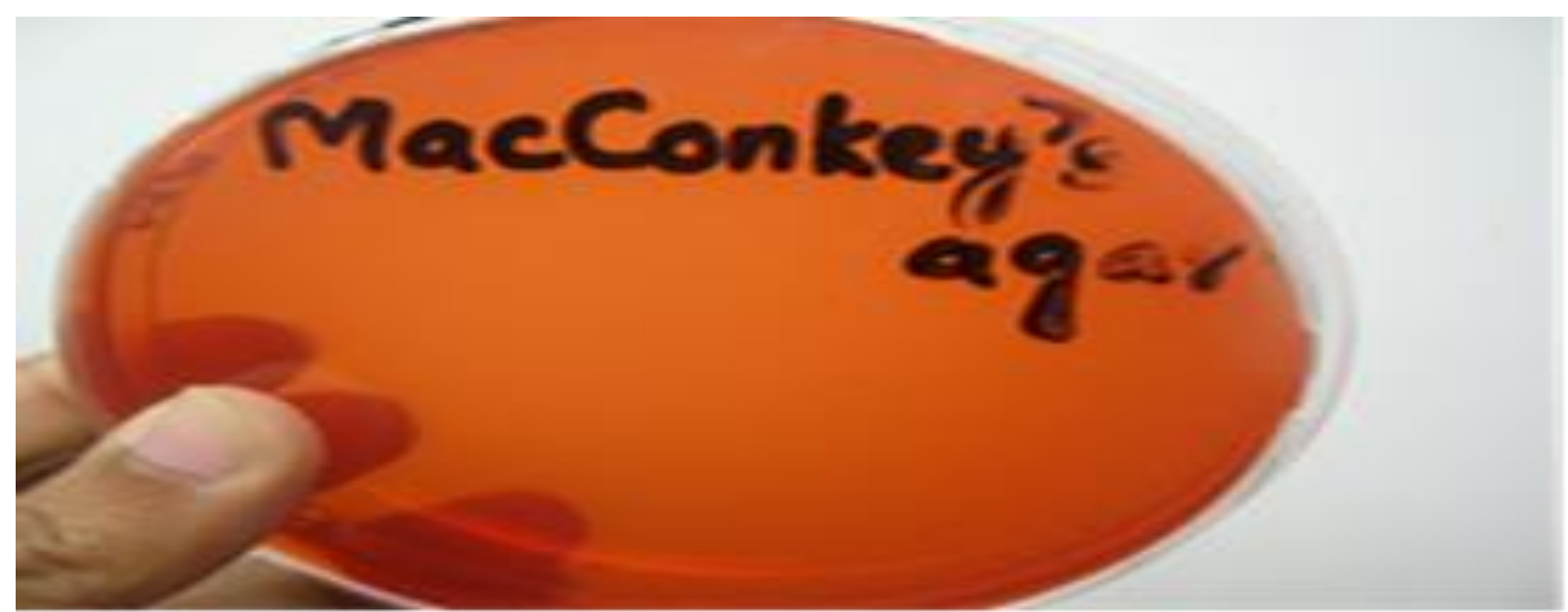

Figure 4. Structure and staining characteristics of $S$. Pneumoniae under microscope

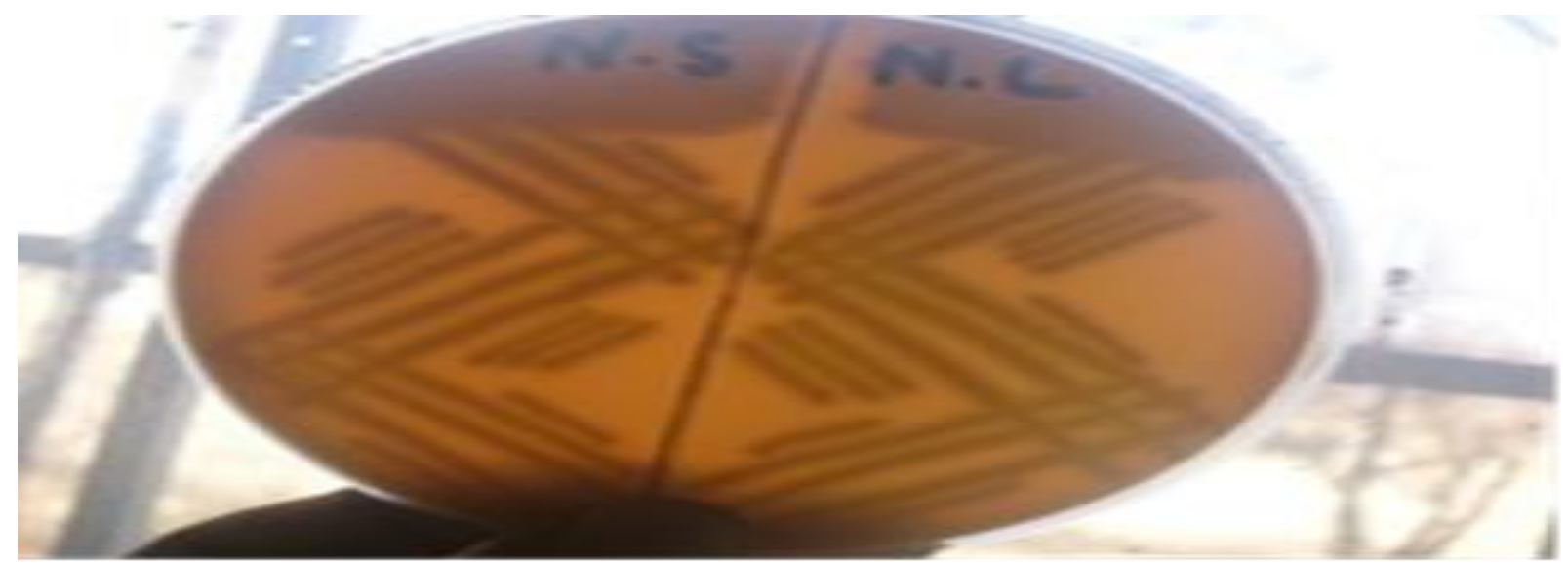

Figure 5. Alpha hemolysis on the blood agar produced by alpha heamolytic strains of $S$. pneumoniae 
Table 1. Different biochemical test and sugar fermentation tests for identification $S$. pneumoniae

\begin{tabular}{|c|c|c|c|}
\hline S. No. & \multicolumn{2}{|c|}{ Biochemical test properties } & S. Pneumoniae \\
\hline 1 & & $\frac{\text { Gram staining }}{\text { Shane }}$ & Gram positive \\
\hline 2 & & Diplococcus \\
\hline 3 & \multicolumn{2}{|r|}{ Motility } & - \\
\hline 4 & \multicolumn{2}{|r|}{ Bile solubility test } & + \\
\hline 5 & & - \\
\hline 6 & \multicolumn{2}{|r|}{ Oxidase test } & - \\
\hline 7 & \multirow{2}{*}{\multicolumn{2}{|c|}{$\frac{6.5 \% \mathrm{NaCl} \text { test }}{\text { TSI Test }}$}} & + \\
\hline 8 & \multirow{2}{*}{\multicolumn{2}{|c|}{$\frac{\text { TSI Test }}{\text { CAMP }}$}} & + \\
\hline 9 & & & + \\
\hline \multirow[t]{2}{*}{10} & \multirow{2}{*}{ 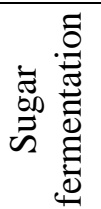 } & Glucose & - \\
\hline & & Raffinose & - \\
\hline 11 & \multicolumn{2}{|r|}{ Optochin disk test } & + \\
\hline 12 & \multicolumn{2}{|r|}{ Bacitacin disk test } & - \\
\hline
\end{tabular}

\section{Discussion}

S. Pneumoniae (pneumococcus) is an essential pathogen of human that causes both delicate upper respiratory infections and serious invasive infections like septicaemia, meningitis and pneumonia. The normal nasopharyngeal microbial flora also contains S. Pneumoniae. The susceptibility of pneumococcal diseases differs in different age groups and different communities $[7,8]$. Several reported risk factors of pneumococcal illnesses are age, race, immunodeficiency, other illness, socioeconomic status, previous antibiotic therapy and day-care [7]. Pneumococcus is a dominant cause of highly lethal and morbid infections [7]. Every year probably one million children expire because of pneumococcal infections and in developing countries most of them are young children [9]. Invasive pneumococcal diseases also exist in developed countries specifically in children and elderly people.

Pneumococcus is a alpha hemolytic, bile soluble, commonly capsulated and grampositive streptococcus. Identification of bacterium is based on the bacteria's colonial morphology on a blood agar plate, optochin sensitivity, bile solubility and the capsule presence. So far 90 different capsular serotypes have been identified [10]. Additionally, in the nasopharynx encapsulated isolates are relatively common. The goal of this research was to isolate the $S$. Pneumoniae from children and adults going through throat infections in different hospitals in Quetta District. Throat swabs and sputum samples were gathered from the youngsters and adults incredibly suffering from throat infection on the base of medical symptoms. When the samples were analyzed on columbia blood agar plates, among (120) samples, (40) isolates produced alpha haemolytic colonies $1-2 \mathrm{~mm}$ in diameter. In growing culture the colonies were appeared in ring shape with flattened centers and raised edges. Alpha heamolysis was produced underneath and in a limited region around the colonies on columbia blood agar following incubation at $37^{\circ} \mathrm{C}$ in $5-10 \% \mathrm{CO} 2$ that were similar as accordance with [11]. On selective media; Crystal Voilet Nalidixic Acid Gentamycin (CVNG) blood agar all (40) isolates produced small, flat, draughtsman type colonies with greenish alpha heamolysis, as described by mast 
group Ltd company. When the colonies from CVNG were streaked on MacConkey agar, no growth had appeared as had described that S. Pneumoniae cannot grow on MacConkey agar. The scientist isolated $48 \%$ S. Pneumoniae from sputum. In our study 40 out of 120 isolates showed positive results that make $30 \%$ of all isolates [12].

Colonies suspected as S. Pneumoniae were confirmed by further processing through a series of biochemical tests. All the isolated colonies were tested for catalase enzyme production by the process used by $[13,14]$ and the tests were negative for catalase production. The isolates were also processed for oxidase test to distinguish organisms on the basis of the presence of a respiratory enzyme, cytochrome. As oxoid (Oxoid Ltd Company) demonstrated S. Pneumoniae are oxidase negative similarly our tests showed negative results for oxidase.

All the isolates were subjected to optochin susceptibility test that resulted in the production of inhibition zones of about 10$14 \mathrm{~mm}$. As demonstrated by [15] that ethylhydrocupreine hydrochloride (optochin) in low concentrations on blood agar would inhibit pneumococcal growth. $S$. Pneumoniae were also tested for bacitracin disc and were found resistant to Bacitracin as described by [6]. The bile solubility tests of the organisms were determined as had reported that Neufeld in 1900 first noticed that bile has the property of being able to dissolve the pneumococcal cell. He proposed this characteristic as an important diagnostic character for discriminate pneumococcus from other streptococci. Neufeld also found that only recently isolated and fatal pneumococcal strains were bile soluble.

Acid production both in slant and butt in triple sugar iron (TSI) test profiles were taken as confirm cases of S. Pneumoniae as had proved by his experiment. The isolates were examined for fermentation of sugar such as glucose and raffinose. All the isolates had utilized glucose both oxidative (without oil) and fermentative (with oil) but could not utilize raffi nose experimentally demonstrated that metabolism of raffinose required "raf cluster" proteins which was not present in all strains of Streptococcus pneumonia. CAMP test was performed to determine the production of CAMP factor and growth of S. Pneumoniae in $6.5 \% \mathrm{NaCl}$ broth was examined and the results were same as [6]. S. Pneumoniae are CAMP negative and do not have the ability to grow in $6.5 \% \mathrm{NaCl}$ broth. The motility of the organisms was determined as described by [16]. This study also showed that Streptococcus pneumonia are non-motile.

The production of hemolysin was determined on the columbia blood agar containing sheep blood as described by [11]. Out of (30) S. Pneumoniae isolates (40) $(30 \%)$ produced alpha haemolysis.

\section{Conclusion}

In fresh culture the colonies were raised but later became flattened with raised edges, giving them a ringed appearance. Alpha heamolysis was produced underneath and zone was narrow around colonies on columbia blood agar following incubation at $37^{\circ} \mathrm{C}$ in $5-10 \% \mathrm{CO}_{2}$. On selective media; Crystal Voilet Nalidixic Acid Gentamycin (CVNG) blood agar all (40) isolates produced small, flat, draughtsman type colonies with greenish alpha heamolysis. When the colonies from CVNG were streaked on MacConkey agar, no growth had appeared.

\section{Authors' contributions}

Conceived and designed the experiments: $\mathrm{R}$ Rasheed \& MK Taj, Performed the experiments: R Rasheed, MK Taj \& K Hasni, Analyzed the data: R Rasheed, MK Taj \& M Shafi, Contributed materials/ analysis/ tools: R Rasheed, MK Taj \& S Rasheed, Wrote the paper: R Rasheed \& MK Taj. 


\section{Acknowledgement}

Present research was conducted at (CASVAB) Centre for Advanced Studies in Vaccinology and Biotechnology University of Balochistan, Quetta.

\section{References}

1. Kenneth T (2011) Todar's Online textbook of bacteriology. Bacterial Protein Toxins.

2. Hussain R (1997). Pneumonia perceptions and management: an ethnographic study in urban squatter settlements of Karachi, Pakistan. Social Sci \& Med 45(7): 991-1004.

3. Lanata CF \& Black RE (2008). Acute lower respiratory infections, in Nutrition and Health in Developing Countries. Springer 179-214.

4. Heiskanen-Kosma (1998). Etiology of childhood pneumonia: serologic results of a prospective, population-based study. The Pediatric Infect Dise $J$ 17(11): 986-991.

5. Austrian R (1986). Some aspects of the pneumococcal carrier state. $J$ of Antimicrobial Chemothera 18(Suppl A): 35-45.

6. Brandi L (2006) Novel tetrapeptide inhibitors of bacterial protein synthesis produced by a Streptomyces sp. Biochem 45(11): 3692-3702.

7. O'Brien KL \& Dagan R (2003). The potential indirect effect of conjugate pneumococcal vaccines. Vaccine 21(1718): 1815-1825.

8. Bogaert D, de Groot R \& Hermans P (2004). Streptococcus pneumoniae colonisation: the key to pneumococcal disease. The Lancet Infect dise 4(3): 144-154.

9. Rajalakshmi B \& Kanungo R (2001). Cost-effective method of serotyping streptococcus pneumoniae using staphylococcal co-agglutination. Indian J of Med Microbiol 19(4): p. 197.

10. Hausdorff WP (2000). Which pneumococcal serogroups cause the most invasive disease: implications for conjugate vaccine formulation and use, part I. Clinical Infect Dise 30(1): 100121.

11. Peled N \& Yagupsky P (1999). Improved detection of Streptococcus pneumoniae in middle-ear fluid cultures by use of a gentamicin-containing medium. J of Clin Microbiol 37(10): 3415-3416.

12. Arora N et al. (2001). Chronic Obstructive Airway Disease in a Hospital Based Study. Indian J of Chest Dise and All Sci 43: 157-162.

13. Buller NB (2004). Bacteria from Fish and Other Aquatic: A Practical Identification Manual. CABI Publising CAB International Wallingford Oxford shire OX108DE. United Kingdom, pp 161.

14. Sawant AA \& Pillai SR (2002). Jayarao, Evaluation of five selective media for isolation of catalase-negative grampositive cocci from bulk tank milk. $J$ of Dairy Sci 85(5): 1127-1132.

15. Morch E (1943). Virulence of the pneumococcus types for mice, p. 157163. In F. A. Richard (ed.), Serological studies on the pneumococci. Einar Munksgaard, Copenhagen, Denmark.

16. Kempf M \& Gravet A (2012). Observatoires regionaux du pneumocoque. Regional pneumococcal observatories: antibiotic susceptibility and serotypes of Streptococcus pneumoniae isolated in France in 2009. Bull Epidemiol Hebd 51(6): 428431. 